\title{
AVALIAÇÃO DA EFICIÊNCIA ENERGÉTICA E DA FORMAÇÃO DE FEO DO FEA DA VSBM*
}

\author{
Lívia Lopes de Oliveira Goulart ${ }^{1}$ \\ Helio Espíndola de Oliveira² \\ Ely da Silva Araujo Junior ${ }^{3}$ \\ André Luiz Vasconcellos da Costa e Silva ${ }^{4}$
}

\section{Resumo}

Com o objetivo de avaliar a estabilidade do processo do FEA 1 da gerência de Aciaria Elétrica da Votorantim Siderurgia unidade Barra Mansa (VSBM) e de entender a eficiência energética e a formação de $\mathrm{FeO}$ no interior do forno, análises foram realizadas durante três meses envolvendo os parâmetros associados a essas variáveis. Foram avaliados consumos de energia elétrica e a energia química, considerando a composição química do mix de sucatas e o uso dos queimadores de gás natural. Notou-se que as condições da escória na etapa de refino não são ideais para a espumação, implicando em maiores perdas térmicas para as paredes do FEA. Para a formação de $\mathrm{FeO}$, foi avaliada a quantidade de coque injetado adicionada e também a relação entre oxigênio e gusa equivalente da carga. Para a melhoria da eficiência energética são propostas melhoria de injeção e adição de insumos de forma que o consumo de energia elétrica possa ser reduzido e o rendimento metálico o maior possível, de forma a garantir um processo que possa manter o produto competitivo no mercado.

Palavras-chave: Energia elétrica; Rendimento metálico; Escória; FEA; Estabilidade operacional.

\section{EVALUATION OF THE ENERGY EFFICIENCY AND FEO FORMATION IN THE VSBM EAF}

\section{Abstract}

In order to assess the process stability of the EAF 1, in Steelmaking Management at Votorantim's Steel Mill and to understand the energy efficiency and FeO formation, the parameters related with these variables were collected and analyzed during three months. Energy consumption, including Electrical and chemical energy based on the chemical composition of the scrap and chemical energy from the natural gas burners were measured. The slag composition indicated that the conditions for foaming were not being achieved and thus, a significant thermal loss to the furnace walls could be expected. For the FeO formation, the amount of injected coke added and also the relationship between oxygen and equivalent load pig iron was evaluated. At the end of the work, some recommendations related to the improvement of the carbon injection and its form are proposed, to achieve a lower electric power input and a high metal yield, to ensure a process that can maintain a competitive position on the market.

Keywords: Electric energy; Metallic yield; Slag; EAF; Operational stability.

1 Engenheira Metalúrgica, Eng. de Processos da Aciaria Elétrica, VSBM, Barra Mansa, RJ, Brasil; livia.goulart.lg1@vsiderurgia.com.br.

2 Facilitador de Rotina FEAs, VSBM, Barra Mansa, RJ, Brasil; helio.oliveira@vsiderurgia.com.br.

3 Engenheiro Metalurgista; M.Eng., Gerente da Aciaria Elétrica, VSBM, Resende, RJ, Brasil; ely.araujo@vsiderurgia.com.br

4 Engenheiro Metalúrgico, PhD, Professor, EEIMVR, UFF, Membro da ABM, Volta Redonda, RJ, Brasil; andre@metal.eeimvr.uff.br. 


\section{INTRODUÇÃO}

Garantir eficiência energética em um Forno Elétrico a Arco (FEA) e manter níveis baixos de $\mathrm{FeO}$ dissolvido na escória são duas práticas importantes na redução de custos de produção das aciarias elétricas. Definir as condições para atingir estes dois objetivos, entretanto, requer elevado conhecimento e controle do processo. Melhorias nestas áreas permitem reduzir os custos, aumentar a produtividade e melhorar a estabilidade do processo, e aprimorar o processo de fabricação dos aços longos de forma a manter o produto competitivo no mercado, garantindo a lucratividade do negócio, e mais que isso, a sobrevivência em períodos de crise.

De acordo com Kirchen et. al [1], as entradas e saídas de energia em um FEA ocorrem de várias formas. A equação 1, apresenta o balanço de energia do FEA proposto por [2]:

$$
\begin{aligned}
E_{\text {Total }}= & \int_{\text {carregamento }}^{\text {varamento }} P_{\text {Elétrica }} \mathrm{d} t+\Delta H_{\text {Oxigênio Injeção }}+\Delta H_{\text {Gás natural - queimadores }} \\
= & \Delta H_{\text {Aço }}+\Delta H_{\text {Esc. }}+\int \Delta \dot{H}_{\text {pós comb! }} \mathrm{d} t+\int \Delta \dot{Q}_{\text {Refrig. }} \mathrm{d} t \\
& +\int \Delta \dot{Q}_{\text {Radiação, outras perdas }} \mathrm{d} t
\end{aligned}
$$

Equação 1

Vários autores [1, 2, 3] discutem os fatores importantes para se atingir a eficiência energética em um FEA. Os fatores mais importantes são listados a seguir:

a) Correta estratificação da carga metálica no interior do FEA, essencial para a fusão uniforme e sem interrupções,

b) Ajuste fino do programa de potência visando tempos e valores corretos de Corrente

(I) e tensão (V) nas etapas de perfuração e fusão da sucata e refino da carga;

c) Ajuste correto do programa químico visando melhor relação $\mathrm{O}_{2}$-gás natural (GN) garantindo mistura perfeita para combustão e vazão supersônica. Este ajuste otimiza a oxidação dos elementos $\mathrm{Si}, \mathrm{C}, \mathrm{P}$, que trarão energia química para o banho através da liberação de calor das reações exotérmicas, listadas na Figura 1.

\begin{tabular}{lll}
\hline & Energy released & \\
\hline Reactions in melt & & \\
$\mathrm{Si}+\mathrm{O}_{2} \rightarrow \mathrm{SiO}_{2}$ & $8.94 \mathrm{kWh} / \mathrm{kg}_{\mathrm{Si}}$ & $11.2 \mathrm{kWh} / \mathrm{m}_{\mathrm{O}_{2}}^{3}$ \\
$\mathrm{Mn}+0.5 \mathrm{O}_{2} \rightarrow \mathrm{MnO}$ & $1.93 \mathrm{kWh} / \mathrm{kg}_{\mathrm{Mn}}$ & $9.48 \mathrm{kWh} / \mathrm{m}_{\mathrm{O}_{2}}^{3}$ \\
$2 \mathrm{Cr}+1.5 \mathrm{O}_{2} \rightarrow \mathrm{Cr}_{2} \mathrm{O}_{3}$ & $3.05 \mathrm{kWh} / \mathrm{kg}_{\mathrm{Cr}}$ & $9.42 \mathrm{kWh} / \mathrm{m}_{\mathrm{O}_{2}}^{3}$ \\
$2 \mathrm{Fe}+1.5 \mathrm{O}_{2} \rightarrow \mathrm{Fe}_{2} \mathrm{O}_{3}$ & $2.05 \mathrm{kWh} / \mathrm{kg}_{\mathrm{Fe}}$ & $6.80 \mathrm{kWh} / \mathrm{m}_{\mathrm{O}_{2}}^{3}$ \\
$\mathrm{Fe}+0.5 \mathrm{O}_{2} \rightarrow \mathrm{FeO}$ & $1.32 \mathrm{kWh} / \mathrm{kg}_{\mathrm{Fe}}$ & $6.58 \mathrm{kWh} / \mathrm{m}_{\mathrm{O}_{2}}^{3}$ \\
$\mathrm{C}+0.5 \mathrm{O}_{2} \rightarrow \mathrm{CO}$ & $2.55 \mathrm{kWh} / \mathrm{kg}_{\mathrm{C}}$ & $2.73 \mathrm{kWh} / \mathrm{m}_{\mathrm{O}_{3}}^{3}$ \\
$2 \mathrm{Al}+1.5 \mathrm{O}_{2} \rightarrow \mathrm{Al}_{2} \mathrm{O}_{3}$ & $5.29 \mathrm{kWh} / \mathrm{kg}_{\mathrm{Al}}$ & $13.84 \mathrm{kWh} / \mathrm{m}_{\mathrm{O}_{2}}^{3}$ \\
$\mathrm{Mo}+\mathrm{O}_{2} \rightarrow \mathrm{MoO}_{2}$ & $1.70 \mathrm{kWh} / \mathrm{kg}_{\mathrm{Mo}}$ & $7.29 \mathrm{kWh} / \mathrm{m}_{\mathrm{O}_{2}}$ \\
$\mathrm{~S}+\mathrm{O}_{2} \rightarrow \mathrm{SO}_{2}$ & $2.75 \mathrm{kWh} / \mathrm{kg}_{\mathrm{s}}$ & $3.94 \mathrm{kWh} / \mathrm{m}_{\mathrm{O}_{2}}^{3}$ \\
$2 \mathrm{P}+2.5 \mathrm{O}_{2} \rightarrow \mathrm{P}_{2} \mathrm{O}_{5}$ & $5.54 \mathrm{kWh} / \mathrm{kg}_{\mathrm{P}}$ & $8.58 \mathrm{kWh} / \mathrm{m}_{\mathrm{O}_{2}}^{3}$ \\
Reactions in gas phase & & \\
$\mathrm{C}+\mathrm{O}_{2} \rightarrow \mathrm{CO}_{2}$ & $9.10 \mathrm{kWh} / \mathrm{kg}$ & $4.88 \mathrm{kWh} / \mathrm{m}_{\mathrm{O}_{2}}^{3}$ \\
$\mathrm{CO}+0.5 \mathrm{O}_{2} \rightarrow \mathrm{CO}_{2}$ & & $7.01 \mathrm{kWh} / \mathrm{m}_{0_{2}}^{3}$ \\
$\mathrm{H}_{2}+0.5 \mathrm{O}_{2} \rightarrow \mathrm{H}_{2} \mathrm{O}$ & & $5.99 \mathrm{kWh} / \mathrm{m}_{\mathrm{O}_{2}}^{3}$ \\
\hline
\end{tabular}

Figura 1: Energia liberada na oxidação dos elementos. Fonte: [2]

Dentro do tópico "eficiência do programa químico" destacam-se ainda, a oxidação do $\mathrm{Si}$, elemento responsável pelo maior ganho em energia, na oxidação, tanto por peso do elemento oxidado como pelo oxigênio consumido, que libera $11,2 \mathrm{kWh} / \mathrm{m}^{3} \mathrm{O}_{2}$ e a 
otimização da pós-combustão (Figura 1), já que a oxidação do $\mathrm{CO}$ a $\mathrm{CO}_{2}$, especialmente, contribui com $7,01 \mathrm{kWh} / \mathrm{m}^{3} \mathrm{O}_{2}$.

d) Controle das perdas de energia e de material (que se traduzem, normalmente, em uma contribuição para a perda de energia), incluindo: redução de perdas para o sistema de refrigeração através da regulagem da água de entrada da carcaça, dos eletrodos, etc; bom controle dos parâmetros de despoeiramento, controlando a pressão do FEA e assim evitando perdas excessivas para os gases e menor perda térmica em forma de radiação através da melhor cobertura do arco elétrico com o uso de uma escória espumante eficiente.

Uma escória espumante efetiva depende, normalmente, de se obter tensão superficial e viscosidade efetiva adequados para se reter $\mathrm{CO}$ pelo tempo necessário na escória $[4,5]$. Estas propriedades, normalmente, são controladas através da basicidade da escória e do teor de fases sólidas presentes, idealmente obtidas pela saturação e magnésiowustita [6]. Pretorious e Oltmann [6] sumarizaram, esquematicamente, o resultado de vários diagramas de solubilidade isotérmica (ISD) e o índice de espumação das escórias, resumindo, semi-quantitativamente, os resultados na Figura 2. É possível verificar nesta figura que, quanto maior é a basicidade, menor será a o teor de $\mathrm{MgO}$ para a saturação e maior o teor de FeO necessário para obter o máximo de espumação.

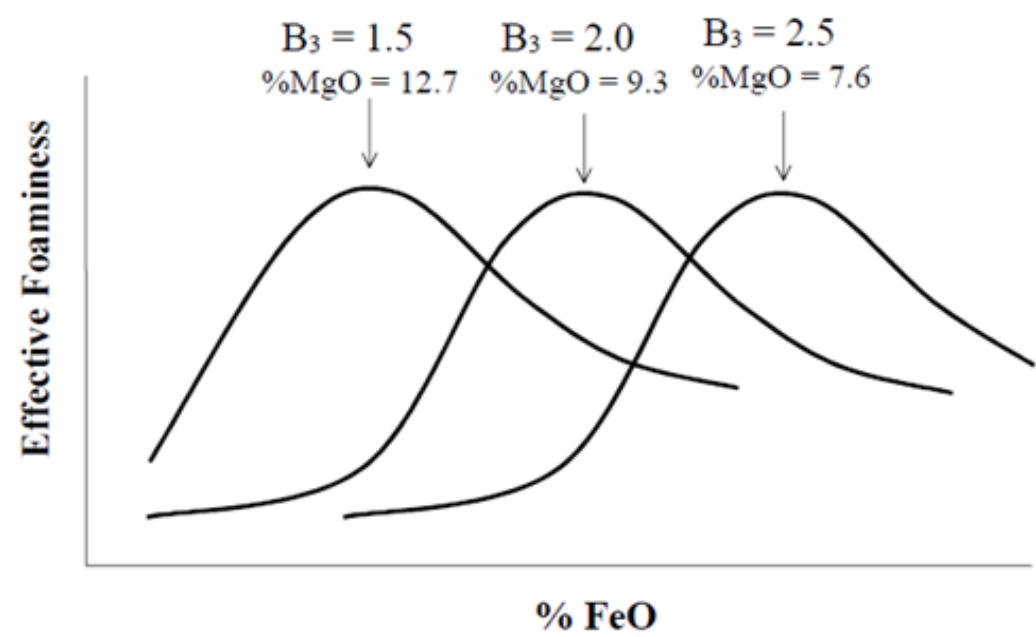

Figura 2: Relação semi-quantitativa inferida entre basicidade ternária $(\mathrm{B} 3=\mathrm{CaO} /(\mathrm{SiO} 2+\mathrm{Al} 2 \mathrm{O} 3)$, solubilidade de $\mathrm{MgO}$ e espumação em função do teor de FeO . Fonte: [6].

A partir da figura 2, também é possível verificar que o $\mathrm{FeO}$ exerce dois papéis importantes na espumação. O primeiro, evidente, pois é o responsável pela formação de gás $\mathrm{CO}$, quando reage com o $\mathrm{C}$ injetado em forma de finos, conforme Equação 2:

$$
(\mathrm{FeO})+1 / 2 \mathrm{O}_{2}=\mathrm{Fe}+\mathrm{CO}
$$

Equação 2

Por outro lado, o FeO tem papel decisivo na solubilidade do MgO ou da magnésiowustita e, portanto, na viscosidade efetiva da escória, essencial para a boa espumação.

A principal fonte de $\mathrm{FeO}$, no FEA, é a oxidação do $\mathrm{Fe}$, em contato com o $\mathrm{O}_{2}$ injetado pelas lanças de oxigênio. Um descontrole consequente de uma baixa otimização do programa químico ou da injeção inadequada de coque podem levar a altos teores desse óxido na escória, o que gera perda de rendimento metálico, reduzindo portanto a competitividade da aciaria. Para Mattielo [7], os teores de FeO para aciarias elétricas 
manterem retenção do $\mathrm{P}$ na escória com bom rendimento metálico devem estar entre 25 e 30\%. Para manter estes níveis de $\mathrm{FeO}$ na escória é essencial controlar, pelo menos os seguintes fatores:

- Relação O2/gusa equivalente (considerando gusa equivalente = gusa + (sucatas de gusa e ferro fundido) ${ }^{\star} 0,78$ ) da carga metálica;

- Momento de início da injeção de coque;

- Forma e local de injeção de coque;

- Granulometria do coque injetado.

Neste trabalho, foram estudadas as condições de eficiência energética do FEA 1 da Votorantim Siderurgia unidade Barra Mansa considerando os aportes de energia Elétrica e química, bem como a formação de FeO. O estudo foi focalizado nas variáveis: relação entre $02 /$ gusa equivalente e tempo e quantidade de entrada de coque injetado, assim como local de injeção e granulometria do coque injetado no FEA.

\section{MATERIAL E MÉTODOS}

O estudo foi realizado no FEA 1 da Gerência de Aciaria Elétrica da Votorantim Siderurgia de Barra Mansa (VSBM). Na VSBM, este forno é o que conta com maior aporte de energia química, incluindo 3 maçaricos pyrejets (sistema multi-função queimador/injetor) dispostos nos pontos frios do FEA e 2 pyrox (sistema queimador), dispostos próximo à porta de escória e ao canal EBT.

Foram analisados dados das corridas processadas no FEA 1 da VSBM em um período de 3 meses consecutivos. Avaliou-se corridas produzidas com os mesmos parâmetros de processo. Avaliou-se o consumo de energia elétrica, oxigênio total, gás natural, mix de carga fria, quantidade de coque injetado e $\mathrm{FeO}$ da escória.

Para o levantamento de energia elétrica, oxigênio total e gás natural, foi utilizado a quantidade computada pelos softwares dos programas de potência e químico, respectivamente.

Para análise do mix de carga fria, foi utilizado o Padrão de Classificação de metálicos da própria Votorantim Siderurgia, onde é estabelecida a composição química média para cada tipo de sucata. A composição média de entrada no mix de carga fria para o período analisado foi a demostrada abaixo:

\begin{tabular}{|c|c|c|c|c|c|c|c|c|}
\hline \multicolumn{9}{|c|}{ Dados de Mix de carga fria } \\
\hline Período/Elemento & $\% \mathrm{C}$ & $\% \mathrm{Mn}$ & $\% \mathrm{Si}$ & $\% \mathrm{P}$ & $\% \mathrm{Cr}$ & $\% \mathrm{Al}$ & $\% \mathrm{Mo}$ & $\% \mathrm{~s}$ \\
\hline Mês 1 & $0,96 \%$ & $0,61 \%$ & $0,35 \%$ & $0,04 \%$ & $0,15 \%$ & $0,02 \%$ & $0,02 \%$ & $0,04 \%$ \\
\hline Mês 2 & $0,97 \%$ & $0,61 \%$ & $0,33 \%$ & $0,04 \%$ & $0,16 \%$ & $0,02 \%$ & $0,02 \%$ & $0,04 \%$ \\
\hline Mês 3 & $0,99 \%$ & $0,61 \%$ & $0,35 \%$ & $0,04 \%$ & $0,15 \%$ & $0,01 \%$ & $0,02 \%$ & $0,04 \%$ \\
\hline
\end{tabular}

Figura 3: Dados de composição química do mix de carga fria. Fonte: Aciaria Elétrica, VSBM.

A quantidade de coque injetado foi levantada de acordo com o apontamento de dados no sistema de informações da GAE - BM. A balança de pesagem do sistema de injeção é testada e calibrada em intervalos regulares, conforme procedimento do fabricante.

É interessante salientar que, a injeção de finos de coque do FEA 1 se deu por 2 pontos, conforme mostra a Figura 4. 


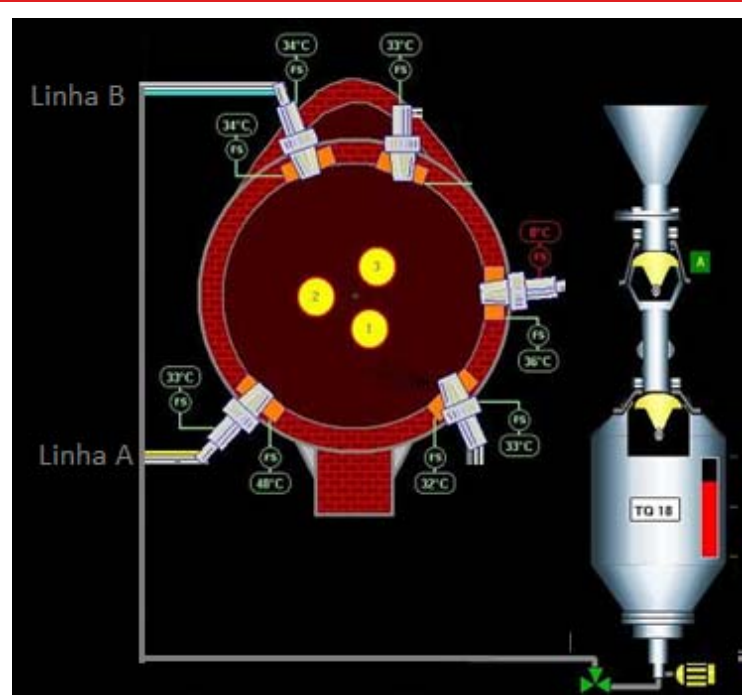

Figura 4: Pontos de Injeção de grafite do FEA 1 (linhas A e B). Fonte: GAE, VSBM.

A taxa de injeção de coque no período foi de $60 \mathrm{~kg} / \mathrm{min}$. O rendimento do coque utilizado garantido pelo fornecedor foi de $87 \%$ de $\mathrm{C}$, considerando seu rendimento no banho de $70 \%$, levando em conta as perdas para o sistema de despoeiramento. A granulometria utilizada foi de $0-6 \mathrm{~mm}$.

A amostra de escória retirada do FEA foi feito seguindo um critério de energia elétrica total para sua retirada do banho. Utilizou-se o valor padrão de $17500 \mathrm{kWh}$, podendo variar entre mais e menos $500 \mathrm{kWh}$ de acordo com o momento de tomada de temperatura do banho.

\section{RESULTADOS E DISCUSSÃO}

\subsection{Resultados}

\subsubsection{Balanço Energético}

Foram estudadas as entradas de energia dos sistemas elétricos e químicos e foi avaliada a efetividade da espumação da escória através da sua composição química, de modo a avaliar o potencial de cobertura do arco elétrico durante a etapa de refino no FEA 1.

Os valores medidos de energia elétrica, oxigênio total e gás natural gastos, juntamente com a composição química do mix de carga fria, possibilitaram calcular as entradas de energia no FEA 1 como apresentado na Figura 5.

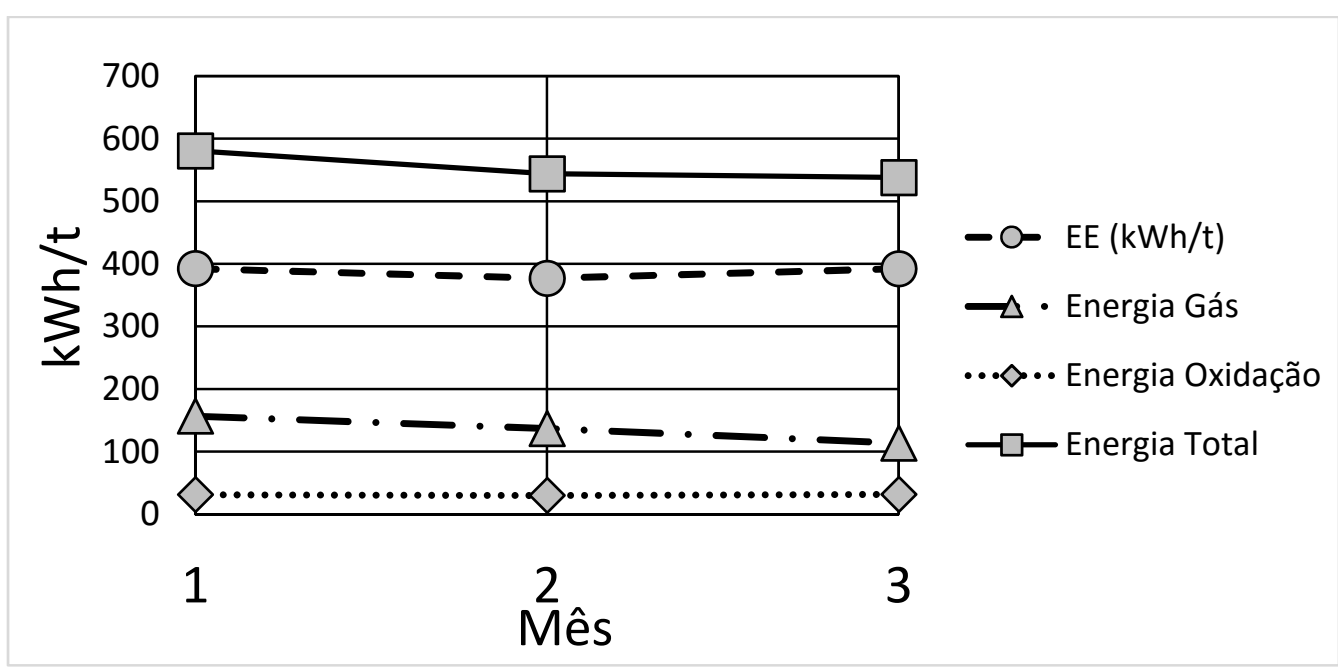

Figura 5: Entradas de energia no FEA 1. Fonte: GAE, VSBM. 
Em função da quantidade de GN empregado, foi calculado o consumo estequiométrico de oxigênio e, adicionalmente, o oxigênio disponível para outras reações no forno, como a oxidação dos elementos contidos na carga. Este valor foi comparado com o oxigênio efetivamente necessário para esta função. Estes resultados são apresentados na Figura 6.

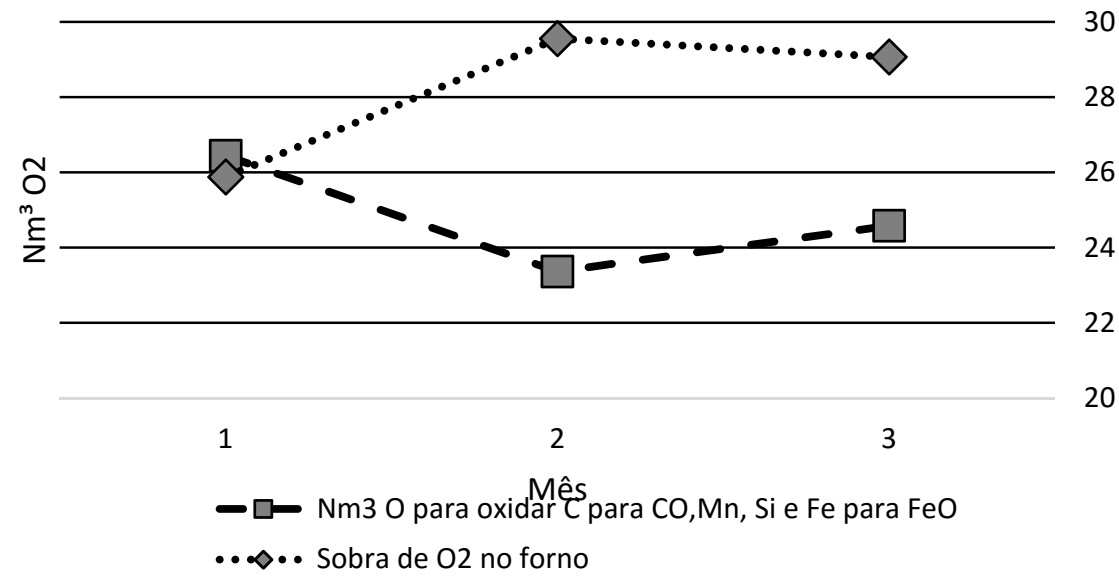

Figura 6: Balanço energético de entrada do FEA 1. Fonte: GAE, VSBM.

A Figura 6 indica que em dois dos três meses estudados, ocorreu considerável excesso de $\mathrm{O}_{2}$ no interior do FEA, o que pode ter ligação com o ajuste do programa químico, levando à perda desse material.

Para se estudar a perda de energia para os painéis e ambiente por meio da irradiação do arco elétrico não coberto, deve-se considerar a espumação da escória. Os diagramas isotérmicos de solubilidade (DIS) [6] são muito empregados nesta estimativa. Nestes diagramas, os dados importantes são a temperatura, basicidade binária, \%MgO e \%FeO, principalmente. A Figura 7 apresenta as composições médias das escórias da VSBM em um diagrama isotérmico de solubilidade para a temperatura e basicidade aproximados para este período:

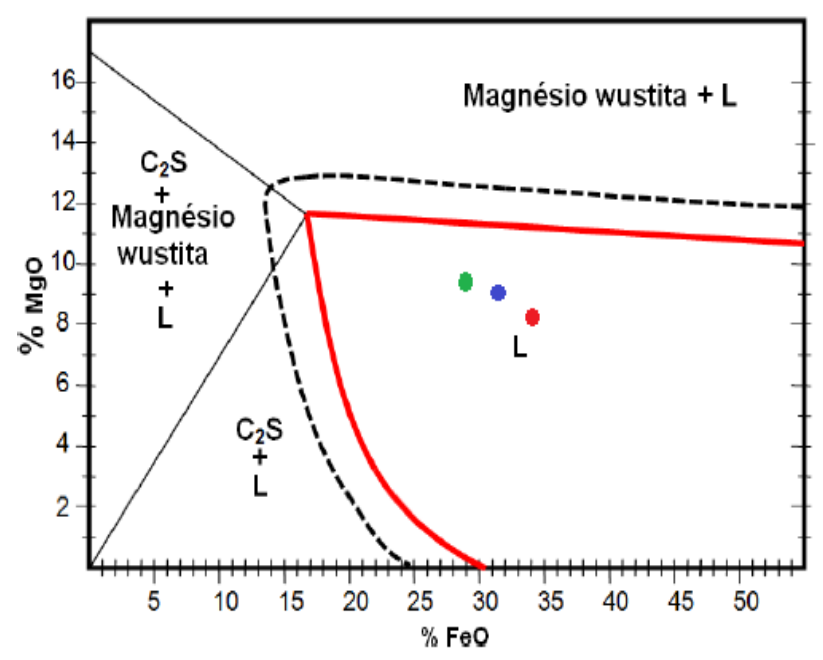

(a)

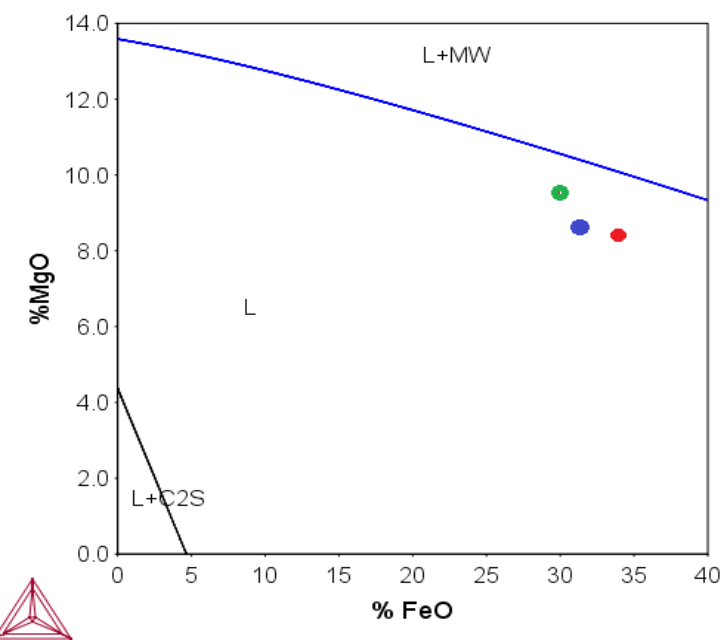

(b)

Figura 7: Localização das escórias da VSBM no diagrama isotérmico de solubilidade para a temperatura e basicidade aproximados para este período. (a) Diagrama de [6]. (b) Diagrama calculado pelo Software Thermo-calc, com as composições completas das escórias. 
A Figura 7 indica que, nos três meses, a escória se localizou no campo da fase líquida do gráfico, o que não é o ideal para a espumação. $O$ balanço energético das perdas para o sistema de refrigeração, escória e radiação não foram calculados neste trabalho.

\subsubsection{Formação de FeO na escória}

A quantidade de $\mathrm{FeO}$ na escória em \% foi levantada com base em amostras retiradas em um momento padronizado no FEA e analisadas através de aparelho Raio-X. Os resultados são apresentados na Figura 8.

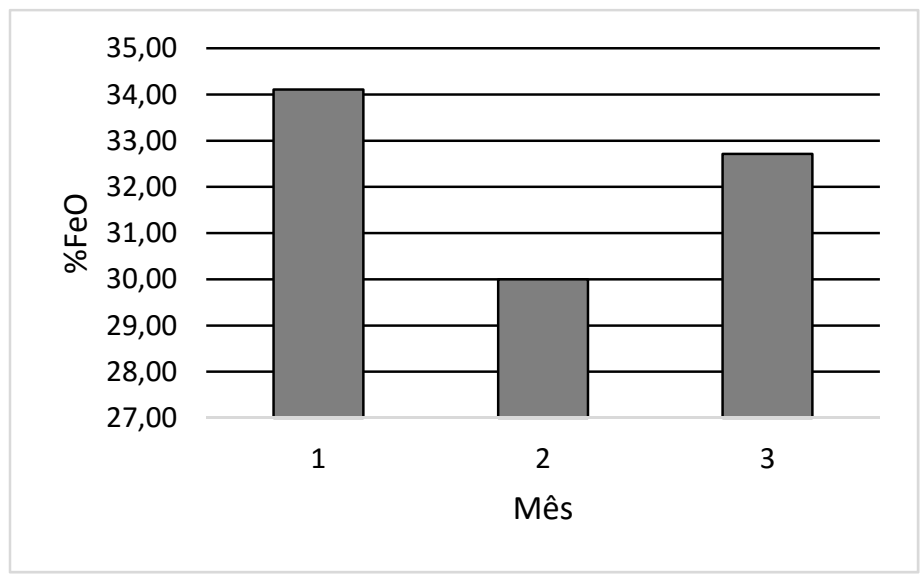

Figura 8: Evolução da percentagem em massa de $\mathrm{FeO}$ das escórias no período estudado. Fonte: GAEVSBM.

Observa-se uma melhora no \%FeO do segundo mês, porém não mantendo esse valor. A média dos três meses foi de $32,7 \%$. As relações entre $\mathrm{O}_{2} /$ gusa equivalente, coque injetado e \%FeO são mostradas na Figura 9:

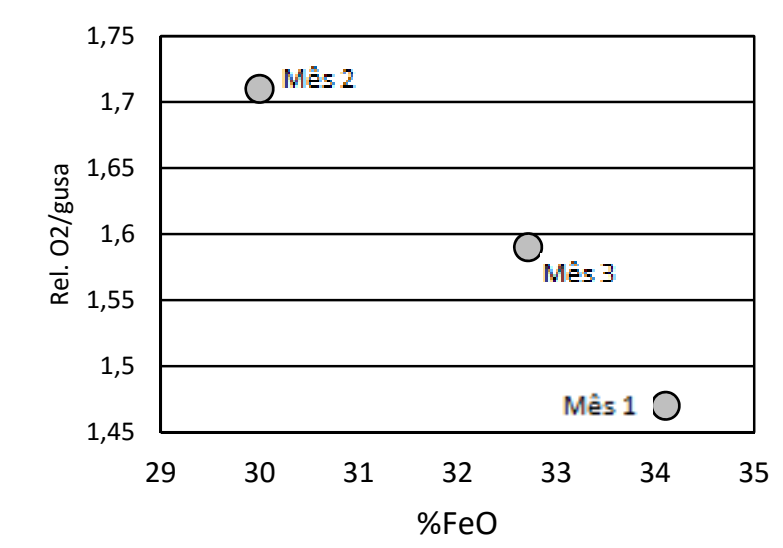

(a)

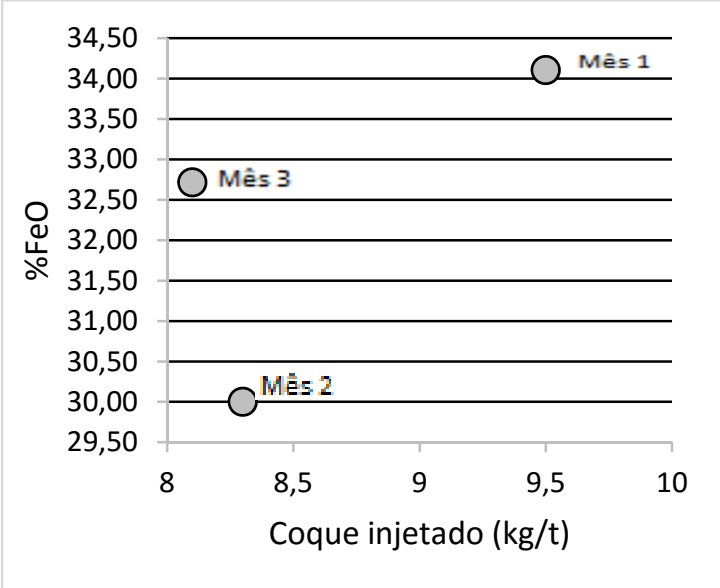

(b)

Figura 9: (a) Relação O2/gusa X FeO, (b) FeO X coque injetado. Fonte: GAE-VSBM.

Observa-se que os dados de coque injetado, não há uma correlação direta com o $\mathrm{FeO}$, porém, a relação O2/gusa equivalente parece seguir uma relação com o \%FeO obtido. 


\subsection{Discussão}

\subsubsection{Balanço Energético}

$\mathrm{Na}$ Figura 5, é possível observar que o consumo de EE tem um comportamento inverso ao aporte de energia através da queima de GN, o que era esperado. É possível avaliar também que a curva de energia de oxidação é bastante uniforme já que o mix de carga também foi uniforme nos três meses, como mostra a Figura 3. Já o desbalanceamento do programa químico é visualizado na figura 6 , onde se observa que em 2 dos 3 meses estudados, há uma sobra considerável de $\mathrm{O}_{2}$ no FEA.

Na figura 7, observa-se que a escória do FEA já no final do refino apresenta aspecto líquido o que é ineficiente para a ocorrência de espumação. Dessa forma perde-se energia elétrica do arco pela sua propagação para os painéis refrigerados e para o ambiente, através de calor, som e luz, com consequente aumento da energia gasta na corrida e redução da eficiência energética do FEA.

Essa perda de energia pode ser representada no gráfico abaixo, quando se compara uma escória convencional com uma escória espumante.

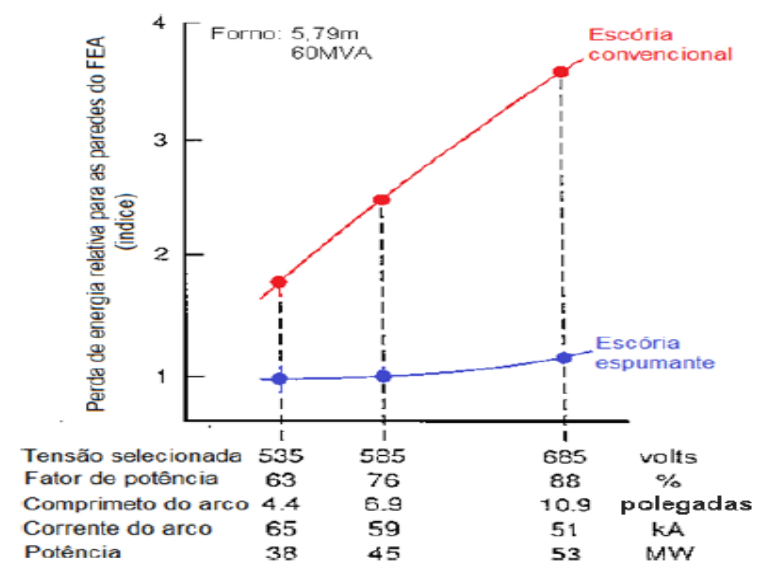

Figura 10: Perdas térmicas relativas para as paredes do forno em função da espumação da escória. Fonte: [8].

Um dos fatores para aumentar a eficiência energética do FEA, é o correto balanceamento da quantidade de oxigênio injetado, evitando perdas e mantendo níveis aceitáveis de oxidação do aço e escória. Além disso, deve-se diminuir a perda térmica para as paredes do FEA. Isto pode ser obtido com a correta formação de espumação da escória. No caso presente, seria necessária a diminuição do \%FeO da escória e o aumento do teor de $\mathrm{MgO}$ para atingir a saturação em \%MgO. Esquematicamente isto corresponde a conduzir a composição da escória para a região entre a linha vermelha e a linha tracejada da Figura 7, onde se teria uma fração de fase sólida adequada para atingir a viscosidade aparente ideal para a espumação. A partir da melhoria desses dois pontos, é possível reduzir as perdas de $\mathrm{O}_{2}$ e de energia no sistema do FEA 1.

\subsubsection{Formação de FeO na escória}

A Figura 9 (a) indica correlação entre o \%FeO e relação O2/gusa equivalente, resultado que não era esperado, visto que quanto mais sobra há de $\mathrm{O} 2$ no FEA, maior seria a \%FeO esperada. Na Figura 9 (b) percebe-se ausência de relação entre \%FeO e coque injetado. Esses dois fatores podem estar relacionados à falta de eficiência do programa químico. Os resultados (Figura 6) indicam que parece haver oxigênio injetado em excesso nos meses 2 e 3, fazendo com que esse insumo se perca no 
sistema do FEA e ambiente. Além disso, é possível perceber que a quantidade de FeO da escória também não se relaciona individualmente com a quantidade de coque injetado, uma vez que no mês que mais se adicionou esse insumo, a \%FeO foi a mais alta, e não o contrário.

Esse fato pode ter ocorrido devido ao rendimento do coque injetado no interior do FEA. O aço no interior do forno é induzido a girar no sentido anti-horário. Isto leva o coque, nas condições de adição atual (figura 4), a chegar rapidamente a porta do FEA, não conduzindo à redução do $\mathrm{FeO}$ e à formação de $\mathrm{CO}$ de forma uniforme. Para melhorar esse aspecto, convém realizar um teste de adição do local mais próximo a porta para o lado oposto, como ilustrado na figura 11.

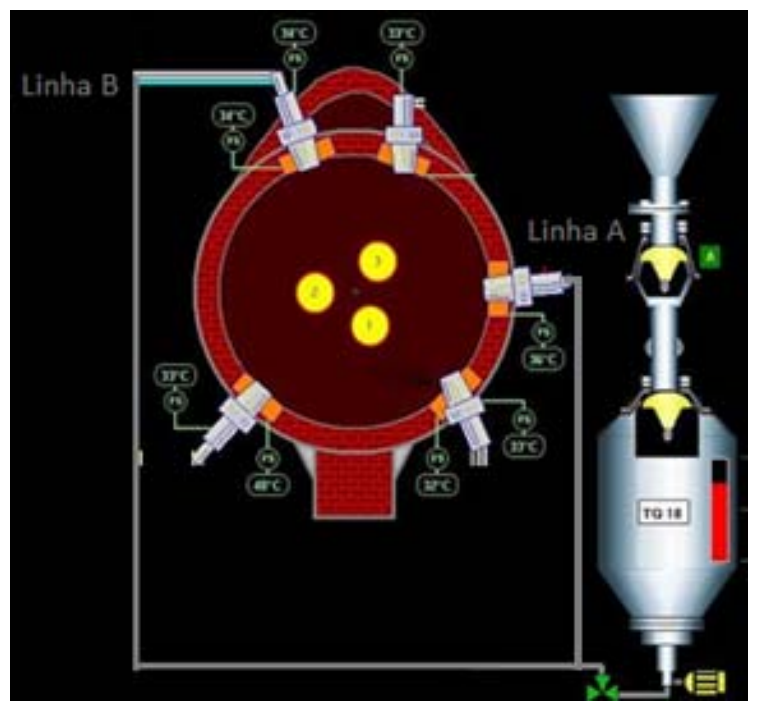

Figura 11: Nova forma de adição de grafite pelas linhas A e B do FEA 1, para teste. Fonte: GAE, VSBM.

Um outro fator que influencia no rendimento metálico é a taxa de injeção de coque. Para uma taxa muito alta, tem-se a rápida saída da escória pela porta do FEA. Considerando que nas condições da VSBM não se trabalha com porta fechada, a escória do início do refino com elevada \%FeO é destinada à baia, não ocorrendo a recuperação do rendimento metálico. Além disso, para uma taxa muito baixa, a cinética da reação de desoxidação é muito lenta, o que não permite recuperar o Fe da escória para o banho. Para esse caso, deve-se ajustar a taxa a um valor entre 70 e $80 \mathrm{~kg} / \mathrm{min}$ e acompanhar a vazão de escória em cada momento do FEA.

Por fim, a perda de rendimento do coque injetado também está ligado à sua perda para o sistema de despoeiramento. Na GAE-BM, essa quantidade, para o FEA 1, chega a 3,5\% no silo da casa de mangas, valor que é considerado elevado quanto comparado ao percentual que se pode encontrar na câmara de combustão. Para minimizar esse efeito, deve-se eliminar ao máximo, os finos de coque que existirem no insumo. Como o valor utilizado atualmente é de $0-6 \mathrm{~mm}$, pretende-se fazer um teste com coque de $2-6 \mathrm{~mm}$.

\section{CONCLUSÕES}

Os resultados apresentados permitem concluir que podem haver melhorias a serem feitas na eficiência energética de entrada do FEA 1 da aciaria elétrica da VSBM. A partir dos dados de $\mathrm{O}_{2}$ foi possível perceber um excesso desse elemento, mostrando que ainda podem ser feitos ajustes no programa químico de forma a aumentar a eficiência do gás natural e também do $\mathrm{O}_{2}$ adicionado. Além disso, foi verificado que a 
escória do FEA no período de refino não era ideal para a cobertura do arco elétrico, o que aumenta as perdas de energia elétrica para o ambiente através de radiação. Para melhorar a espumação da escória deve-se diminuir o FeO do banho além de aumentar a basicidade binária da escória e a saturação em MgO.

Em relação à formação de $\mathrm{FeO}$, percebe-se que os valores não se correlacionam, como seria esperado, com os parâmetros $\mathrm{O}_{2}$ /gusa equivalente ou quantidade de coque injetado. Isto também indica a necessidade de ajuste do programa químico e a necessidade de melhor controle das diversas perdas de coque que podem estar ocorrendo, resultando em aproveitamento inferior ao valor indicado de $70 \%$. Para realizar essa avaliação e melhorar o rendimento metálico do banho, alguns testes foram propostos:

- Alteração dos locais de injeção de coque (maior rotação no banho);

- Alteração da taxa de adição;

- Alteração da granulometria do coque (menor perda para o despoeiramento). A partir dessas alterações, espera-se obter melhor rendimento metálico e melhor viscosidade da escória de FEA, possibilitando uma maior eficiência energética.

\section{Agradecimentos}

À Escola de EEIMVR e à Votorantim Siderurgia Barra Mansa pelo apoio e suporte técnico.

\section{REFERÊNCIAS}

1. Marcus Kirschen et. Al. Energy efficiency and the influence of gas burners to the energy related carbon dioxide emissions of electric arc furnaces in steel industry. 2009 Disponível em https://www.researchgate.net/publication/245076796, [acesso em 20/04/2016]

2. Luis R. Jaccard. Curso de Fornos Elétricos a Arco. 2009. Seminário de Aciaria ABM. São Paulo, SP.

3. Alexandre Libório John. Melhorias no desempenho dos injetores supersônicos em operação em um FEA. 2009. Dissertação apresentada ao Curso de Pós Graduação em Engenharia Metalúrgica, UFRGS.

4. Ito K, Fruehan RJ. Study on the foaming of $\mathrm{CaO}-\mathrm{SiO} 2-\mathrm{FeO}$ slags: Part I. Foaming parameters and experimental results. Metallurgical Transactions B. 1989;20B (August):50914.

5. Ito K, Fruehan RJ. Study on the foaming of CaO-SiO2-FeO slags: Part II. Dimensional analysis and foaming in iron and steelmaking processes. Metallurgical Transactions $B$. 1989;20B (August):515-21.

6. E Pretorious, H Oltmann. Fundamentals of the EAF Process. LWB Refractories; 2011, disponivel em http://etech.Iwbref.com/Downloads/ Theory/

Fundamentals\%20of\%20the\%20EAF\%20Process.pdf. [acesso em 04 abr 2016]

7. Jakson Marttiello. Estudo das causas de variabilidade dos teores de FeO da escória de um FEA. Trabalho de diplomação apresentado ao Departamento de Metalurgia da escola de Engenharia da UFRGS.

8. Thiago Augusto Ávila. Condicionamento de escórias em Forno Elétrico a arco para otimização das condições de espumação da escória e refino do aço. 2011. Dissertação apresentada ao Curso de Pós graduação em Engenharia Metalúrgica da escória de Minas, UFMG. 